\title{
Proceedings of the 27th ACM International Conference on Information and Knowled! Management
}

\begin{tabular}{|c|c|c|}
\hline General Chairs: & Alfredo Cuzzocrea & University of Trieste, Italy \\
\hline rogram Chairs: & James Allan & University of Massachusetts, USA \\
\hline & Norman Paton & University of Manchester, United Kingdom \\
\hline & Divesh Srivastava & AT\&T Labs Research, USA \\
\hline & Rakesh Agrawal & Data Insights Lab, USA \\
\hline & Andrei Broder & Google Research, USA \\
\hline & Mohammed Zaki & Rensselaer Polytechnic Institute, USA \\
\hline & Selcuk Candan & Arizona State University, USA \\
\hline & Alexandros Labrinidis & University of Pittsburgh, USA \\
\hline & Assaf Schuster & Technion, Israel \\
\hline & Haixun Wang & Google Research, USA \\
\hline
\end{tabular}

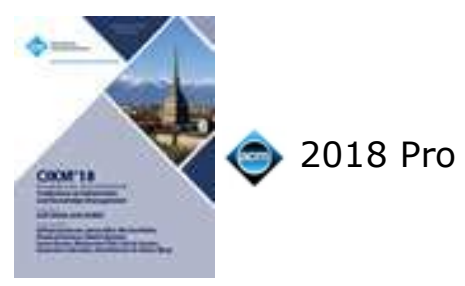

Bibliometrics

Citation Count: 9

Downloads (cumulative): 37

Downloads (12 Months): 37

Downloads (6 Weeks): $4,9 \mathrm{C}$

Publication of:

- Conference

CIKM '18 The 27th ACM International Conference on Information and Knowledge Management

Torino, Italy - October 22 - 26, 2018

ACM New York, NY, USA @2018

\section{Contact Us | Switch to single page view (no tabs)}

\begin{abstract}
\begin{tabular}{|c|c|c|c|c|c|c|c|}
\hline Abstract & Source Materials & Authors & References & Cited By & Index Terms & Publication & Reviews \\
\hline
\end{tabular}
It is our great pleasure to welcome you to the 27th ACM International Conference on Information and Knowledge during October 22-26, 2018 in Turin, Italy. The CIKM 2018 theme is "From Big Data and Big Information to Big K the emerging research trend on big data. Indeed, strategically positioned at the intersection of research on the $\mathrm{r}$ information, and data, CIKM is uniquely situated to highlight technologies and insights that truly materialize the $\mathrm{E}$ Knowledge vision of the future. This year's CIKM continues its tradition of being the premier forum for presentatic reports on leading edge issues of knowledge management, information retrieval, and databases. Along this line, 1 practitioners a unique opportunity to share their perspectives with others interested in the various aspects of the! The call for papers attracted submissions from all over the world. Statistics deriving from the program committee Like in its tradition, CIKM 2018 comprises the following three keynote speakers, whose valuable and insightful tal understanding of the future:

Shifting Information Interactions, Maarten de Rijke (University of Amsterdam, Netherlands);

Teaching Artificial Agents to Understand Language by Modelling Reward, Edward Grefenstette (DeepMind and Uni Kingdom);

Alexa and her Shopping Journey, Yoelle Maarek (Amazon, Israel).

The complete program is organized in 5 parallel sessions, where full research papers, short research papers, dem papers are presented. The program also includes 8 tutorials on timely research topics and 9 co-located workshop from data and text mining in biomedical informatics to entity retrieval and knowledge-driven analytics, and so for Like in the past edition, CIKM 2018 hosts CIKM AnalytiCup, an innovative event where research teams from all or life analytical problems posed by our corporate sponsors, Alibaba. The finalists for the challenge competition part showdown in front of corporate judges who elect the final winner.
\end{abstract}


The ACM Digital Library is published by the Association for Computing Machinery. Copyright (c) 2019 ACM, Inc. Terms of Usage Privacy Policy Code of Ethics Contact Us 